Single transduction channel openings have been measured in hair cells, but only with difficulty: the number of active channels must be reduced drastically by a transient exposure to micromolar levels of calcium ${ }^{6}$. Transduction is abolished irreversibly by the treatment. The experiments described by Assad et al. provide an explanation for this manipulation by showing that the tip links can be removed physically by treating the bundle with very low levels of calcium (using BAPTA as a chelator for 10 seconds). More remarkably still, the

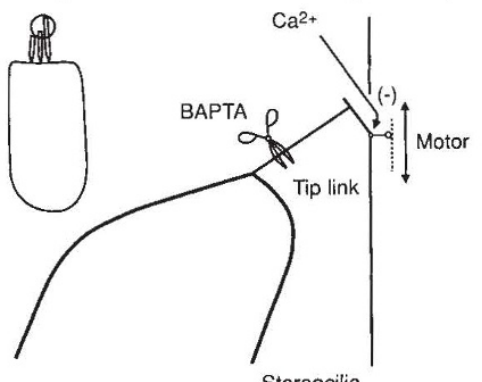

A model of hair cell transduction in the stereocilia (site circled on hair cell in the inset). The tip links run between the top of one stereocilium and its neighbour, where the tensioning motor could be sited. The channel may be at either end of the link. A route for calcium entry is shown. The links are severed by BAPTA ${ }^{1}$.

bundle moves slightly (by $10-100 \mathrm{~nm}$ ) towards the tallest stereocilium as though the bundle had previously been under tension. These observations show that there are ways of altering components of the bundle very selectively.

The question of whether the links are directly coupled to the channel or to some other intermediate structure remains unclear. Is it possible that the calcium-sensitive tip links do nothing more than simply hold the bundle together, and that the mechano-electric transduction step occurs where adjacent membranes of the stereocilia come into contact? Oocyte stretch-activated channels $^{7}$, the hair-cell transducer and apical membrane channels from epithelial cells are sensitive to amiloride, which blocks channels in a voltage-dependent manner. Surprisingly, there is an antibody which binds to the latter two but which does not necessarily co-localize to either end of the hair-cell tip link ${ }^{8}$. This might even indicate that there are more channels in the apical membrane than those associated with the tip links.

In hair cells, channels opened by bundle movement eventually close (although they can be reopened by further displacement), such adaptation depending upon the internal concentration of calcium $^{9,10}$. So what is the interaction between calcium and the tip link? In order to re-exert a force on the channel after a steady displacement, the link needs either to shorten or to reposition itself along the stereocilia. One proposal is that a molecular motor placed at one end of the link pulls the filament tight ${ }^{5}$. Even though there are suggestions that the motor may be fuelled by ATP ${ }^{11}$, it would have to possess some curious properties: it would need to run continuously but to slip, and to slip more with increased levels of internal calcium in order to reduce link tension. Transmembrane sliding-motor systems are known in flagella ${ }^{12}$, but are not as yet described in sensory cells.

The other explanation for hair-cell adaptation is that the transducer channel is itself closed by the calcium which enters. This would form a feedback loop tightly regulating the activity of the channel $^{10}$. But although this model accounts for many features, it does not explain the loss of transduction under micromolar calcium conditions. The problem with the tip-link model is that it predicts changes in bundle stiffness not seen during experimentally controlled adaptation $^{10}$. There are thus features of the transduction process which neither explanation can entirely account for.

Assad et al. nonetheless describe the first observed relationship between bundle morphology and transduction currents since hair-cell signals were shown to be produced by deflection of the stereocilia of the hair bundle ${ }^{13}$. Their measurements of bundle motion may even hint at a rapid tensioning of the links, before they snap. It should make matters much clearer when the forces in the tip links are measured directly. The next stage is to dissect the components of the transducer channel to determine its precise molecular environment. The problem is that there are only about 100 channels in each hair cell: now, there's a challenge for molecular biology.

Jonathan Ashmore and Michael Evans are in the Department of Physiology, School of Medical Sciences, University of Bristol, Bristol BS8 1TD, UK.

1. Assad, J. A., Shepherd, G. M. G. \& Corey, D. P. Neuron 7. 985-994 (1991).

2. Jaramillo, F. \& Hudspeth, A. J. Neuron 7, 409-420 (1991).

3. Ohmori, H. J. Physiol. 399, 115-138 (1988)

4. Pickles, J. O. et al. Hearing Res. 15, 103-112 (1984).

5. Howard, J., Roberts, W. A. \& Hudspeth, A. J. A. Rev. Biophys. biophys. Chem. 17, 99-124 (1988).

6. Crawford, A. C., Evans, M. G. \& Fettiplace, R. J. Physiol. 434, 369-398 (1991).

7. Lane, J. W., McBride Jr, D. W. \& Hamill, O. P. J. Physiol. 441, 347-366 (1991)

8. Hackney, C. M. \& Furness, D. N. J. Physiol. 438, 124P (1991).

9. Assad, J. A., Hacohen, P. \& Corey, D. P. Proc. natn Acad. Sci. U.S.A. 86, 2918-2922 (1989)

10. Crawford, A. C., Evans, M. G. \& Fettiplace, R. J. Physiol. 419, 405-434 (1989).

11. Gillespie, P. G. \& Hudspeth, A. J. Biophys. J. 61, 516a (1992).

12. Bloodgood, R. A. in Prokaryotic and Eukaryotic Flagella (eds Amos, W. B. \& Duckett, J. G.) 353-380 (Cambridge University Press, 1982)

13. Hudspeth, A. J. \& Jacobs, R. A. Proc. natn. Acad. Sci. U.S.A. 76. 1506-1509 (1979).

\section{Evil spirits}

THE hangover, that stern natural punishment for alcoholic overindulgence, is blamed not on alcohol itself, but on the various chemical 'congeners' present in most alcoholic drinks. Aldehydes, tannins, acids and higher esters are the chief villains. They are most plentiful and ferocious in chemically complex drinks like brandy and red wine. Those who mix their drinks, and thus get a richer assortment of congeners, are said to suffer worst.

Daedalus is pondering these strange facts. Congeners, he notes, cannot make up more than a few parts per thousand of any drink - yet they can temporarily induce a degree of malaise and wretchedness normally experienced only by the seriously ill. More wonderful still, they do it after about an eight-hour delay. DREADCO chemists are therefore scouring the market for the cheapest red plonk, the crudest brandy and the most debased 'firewater' whiskies, and are extracting their congeners. Mixed together and concentrated to about a ten per cent solution, the congeners will form a subtle and horrific liquor. The hapless consumer won't even get drunk. But a few hours later he will get the father and mother of all hangovers.

With a little chemical cunning "Headsplitter" (as this fearsome beverage is known among its creators) may even be made quite palatable. For the flavour and character of a drink are also due to its congeners. The DREADCO team is being supervised by a sadistic winemaster, who hopes to endow Headsplitter with a rich and appealing bouquet.

The plan, of course, is to market Headsplitter as a classic form of aversion therapy for otherwise unreformable alcoholics. The patient (victim might be a better word) will be offered a generous glass of the product; a few hours later, when it is just about to strike, he will be given a little of his favourite tipple. The devastating effect, and its apparent cause, should make an indelible impact on even the most dedicated connoisseur.

Headsplitter may find wider markets. Many states impose only four criminal punishments: fine, imprisonment, death, and confiscation of driving licence. Headsplitter could provide a fifth sanction. Cheap, easily calibrated and administered, and doing no lasting harm, it could transform jurisprudence. Yet Daedalus suspects that even this grisly deterrent may acquire devotees of its own. One theory of alcoholism holds that it is a self-destructive 'game' whose unacknowledged payoff is the hangover. Headsplitter will deliver a payoff of unparalled intensity, and a black market may develop in the product. David Jones 